\title{
CRECIMIENTO Y CARACTERÍSTICAS POSTCOSECHA DE FRUTOS DE GENOTIPOS NATIVOS DE TOMATE (Solanum lycopersicum L.)
}

\section{FRUITS GROWTH AND POSTHARVEST CHARACTERISTICS OF NATIVE TOMATO GENOTYPES (Solanum lycopersicum L.)}

\author{
Fabiola Pérez-Díaz' ${ }^{1}$ Ma. de Lourdes Arévalo-Galarza ${ }^{*}$, Laura J. Pérez-Flores ${ }^{2}$, \\ Ricardo Lobato-Ortiz ${ }^{1}$ y Martha E. Ramírez-Guzmán ${ }^{3}$
}

\begin{abstract}
'Colegio de Postgraduados, Programa de Recursos Genéticos y Productividad, Montecillo, Texcoco, Estado de México, México. ${ }^{2}$ Universidad Autónoma Metropolitana, Unidad Iztapalapa, Ciudad de México, México. ${ }^{3}$ Colegio de Postgraduados, Programa de Estadística, Montecillo, Texcoco, Estado de México, México.
\end{abstract}

*Autor de correspondencia (larevalo@colpos.mx)

\section{RESUMEN}

El tomate (Solanum lycopersicum L.) es una de las hortalizas más importantes en el mundo, con un consumo per capita de $20.2 \mathrm{~kg}$. México es considerado como centro de domesticación de esta especie y cuenta con amplia diversidad morfológica de frutos, como los tipos riñón, cereza, ojo de venado (OV), chino criollo (CC), pera, bola y saladette, además de variabilidad genética en composición química, resistencia a plagas, enfermedades y adaptación a condiciones extremas de cultivo. Los materiales nativos pueden ser usados en programas de mejoramiento genético para el desarrollo de nuevas variedades con características que se han perdido en las variedades comerciales. El objetivo de este estudio fue evaluar el crecimiento y variables fisicoquímicas relacionadas con la calidad de fruto de ocho genotipos de tomate nativos de Veracruz y Puebla, México, además de un híbrido experimental (CP-HER) y un híbrido comercial (CID-HC), clasificados en cinco grupos (cereza, OV, CC, riñón y saladette), en los cuales se midió el crecimiento desde el amarre hasta la cosecha. Los frutos se cosecharon en estado rompiente (Estado 1) y rojo (Estado 4) y se evaluaron a los 0,7 y 14 días de almacenamiento. Las variables evaluadas fueron pérdida de peso, color, firmeza, sólidos solubles totales, acidez titulable y azúcares. Los resultados mostraron que los frutos más precoces en alcanzar su tamaño máximo y madurez de consumo fueron los de tipo cereza LOR88 a los 59 días después de floración. Los frutos del genotipo CC LOR102 presentaron mayor firmeza inicial, pero mostraron un descenso significativo durante el almacenamiento, además de ser susceptibles al ataque de hongos, junto con los frutos tipo riñón. Los genotipos con potencial para ser utilizados como fuente de germoplasma para incrementar el dulzor fueron riñón LOR77 y LOR119; los de mayor vida de almacenamiento fueron los pertenecientes al tipo cereza y la mayor firmeza la presentaron CC LOR102 y riñón LOR82.

Palabras clave: Calidad postcosecha, contenido de azúcares, crecimiento, índice de madurez.

\section{SUMMARY}

Tomato (Solanum lycopersicum L.) is one of the most important vegetables in the world, with a per capita consumption of $20.2 \mathrm{~kg}$. Mexico is considered as a domestication center of this species and has a wide morphological diversity of fruits, such as kidney-shape, cherry, deer eye (OV), creole chinese (CC), pear, globe-shape, and saladette types, as well as genetic variability in chemical composition, pests and diseases resistance and adaptation to extreme growing conditions. Native materials can be used in breeding programs for the development of varieties with characteristics that have been lost in commercial varieties. The objective of this study was to evaluate the growth and physicochemical traits related with fruit quality of eight tomato genotypes native to Veracruz and Puebla, México, along with an experimental hybrid (CP-HER) and a commercial hybrid (CID-HC), classified into five groups (cherry, OV, CC, kidney and saladette) in which growth was measured from fruit set to harvest. Fruits were harvested in the breaking stage (Stage 1 ) and red stage (Stage 4) and evaluated at 0,7 and 14 days under storage. The traits evaluated were weight loss, color, firmness, total soluble solids, titratable acidity and sugars. Results showed that the earliest fruits to reach their maximum size and ripening of consumption were those of cherry type LOR88 at 59 days after flowering. Fruits of the CC LOR102 genotype showed greater initial firmness, but showed a significant decrease during storage, in addition to being susceptible to fungal attack along with the kidney-type fruits. Genotypes with potential to be used as a source of germplasm to increase sweetness were kidney LOR77 and LOR119; the ones with the longest shelf life were those belonging to the cherry type, and those with the greatest firmness were CC LOR102 and kidney LOR82.

Index words: Growth, maturity index, postharvest quality, sugar content.

\section{INTRODUCCIÓN}

El tomate (Solanum lycopersicum L.) pertenece a la familia de las solanáceas, es originario del oeste de América del Sur, entre el norte de Chile y Ecuador y posteriormente fue distribuido hacia el trópico y subtrópico de México, país considerado centro de domesticación (Peralta et al., 2006). El consumo per capita se ha incrementado constantemente, de $15.4 \mathrm{~kg}$ en 2001 a $20.2 \mathrm{~kg}$ en 2011 a nivel mundial; en Estados Unidos de América se ubica en $41 \mathrm{~kg}$. En México, el consumo per capita es de $15 \mathrm{~kg}$ por año y las variedades más consumidas son saladette, bola y cereza o cherry (FIRA, 2016).

Las variedades de tomate presentan una amplia diversidad de tamaños, formas y colores de frutos, los cuales se han clasificado en grupos llamados riñón, cereza, 
ojo de venado (OV), chino criollo (CC) o cuadrado, pera, bola, saladette, los cuales presentan amplia variabilidad genética (Bonilla-Barrientos et al., 2014; Vásquez-Ortiz et al., 2010); no obstante, se estima que $80 \%$ de las colectas realizadas a nivel mundial no ha tenido ningún tipo de caracterización (Marín-Montes et al., 2016).

El tomate cultivado ha tenido un proceso de domesticación y selección con el fin de obtener mayor rendimiento, frutos uniformes en tamaño, mayor firmeza y vida de anaquel (Carrillo-Rodríguez et al., 2013); ésto ha provocado que el cultivo tenga menos de $5 \%$ de la variación genética que presentan los genotipos silvestres, lo que los hace susceptibles a factores abióticos (Bai y Lindhout, 2007), los cuales representan un gran reto ante el cambio climático; de la misma manera, en el caso de los factores bióticos, Nieto-Ángel et al. (2019) mencionan que en los últimos años se han desarrollado nuevas enfermedades. Florido et al. (2016) reportaron dos cultivares de México con resistencia al estrés hídrico, mientras que EstradaTrejo et al. (2014) encontraron ocho poblaciones de tomates nativos de Puebla y Veracruz, México tolerantes a la salinidad del suelo; asimismo, Álvarez-Hernández et al. (2009) reportaron incidencia baja de insectos fitófagos que se consideran plagas en tomate, en poblaciones silvestres de tres regiones de Michoacán.

En este contexto, se requiere encontrar materiales que contribuyan a mejorar el sabor y otras propiedades de calidad de los frutos comerciales, ya que son características que se han descuidado entre los criterios de selección que el consumidor demanda (Figàs et al., 2015). George et al. (2004) y Juárez-López et al. (2009) encontraron genotipos nativos que presentan mayor acidez (0.50 - $1.01 \%)$ que los híbridos $(0.32-0.72 \%)$. En frutos de siete cultivares del sur de España se reportaron contenidos de glucosa entre 8.5 y $10 \mathrm{mg} \mathrm{g}^{-1}$ y de fructosa entre 9.6 y $12.4 \mathrm{mg} \mathrm{g}^{-1}$, además de un contenido de ácido cítrico de 322 a 389 mg $100 \mathrm{~g} \mathrm{~g}^{-1}$ (Hernández et al., 2008). Estos estudios indican que los genotipos silvestres tienen amplia diversidad y son fuente importante de germoplasma para el mejoramiento de la calidad fisicoquímica de frutos.

Por lo anterior, el objetivo de este estudio fue evaluar el crecimiento y algunas variables asociadas con la calidad fisicoquímica de frutos de genotipos nativos, un híbrido experimental y un híbrido comercial de tomate como testigos, que permita comparar las características de los frutos de interés para el mejoramiento de esta especie.

\section{MATERIALES Y MÉTODOS}

\section{Material vegetal}

Se evaluaron ocho genotipos nativos de tomate (LOR52, LOR88, LOR16, LOR18, LOR102, LOR82, LOR119 Y LOR77) provenientes de los estados de Puebla y Veracruz, México (Bonilla-Barrientos et al., 2014), un híbrido experimental (CP-HER) y un híbrido comercial (CID-HC) como testigos. Los materiales fueron clasificados en cinco grupos por las características morfológicas de los frutos (Figura 1). Los grupos son: cereza, ojo de venado (OV), chino criollo (CC), riñón y saladette. Las semillas fueron proporcionadas por el Programa de Conservación y Mejoramiento de los Recursos Genéticos del Jitomate del Colegio de Postgraduados.

\section{Manejo del cultivo}

Durante el ciclo primavera-verano (abril-agosto) 2016, las semillas de los materiales se sembraron en charolas con peat moss y se colocaron en un invernadero a $17.8 \pm 4.3^{\circ} \mathrm{C}$ y $79.7 \pm 12.9 \%$ HR ubicado en Montecillo, Texcoco, México (19³0' N y $98^{\circ}$ 53' O) a 2250 msnm. El transplante se realizó a los 30 días después de la siembra en bolsas de polietileno con tezontle rojo. El riego se realizó con solución nutritiva de Steiner (Steiner, 1984) al $50 \%$ en la etapa vegetativa y al $100 \%$ durante la floración y fructificación. Las plantas se condujeron a un solo tallo y se removieron los brotes laterales. Como medida preventiva se realizó una aplicación de los plaguicidas Ampligo® (2 $\left.\mathrm{mL} \mathrm{L}^{-1}\right)$ y Beleaf ${ }^{\circledR}\left(1 \mathrm{~g} \mathrm{~L}^{-1}\right)$ y los fungicidas Ridomil Gold ${ }^{\circledR}$ $\left(6 \mathrm{~mL} \mathrm{~L}^{-1}\right)$ y Amistar ${ }^{\circledR}\left(1.5 \mathrm{~g} \mathrm{~L}^{-1}\right)$ durante el crecimiento de los frutos. Los frutos se cosecharon en dos diferentes estados de madurez (USDA, 2005), 59 d después de la floración (agosto de 2016), a partir del tercer racimo y se almacenaron a temperatura ambiente $\left(23.5 \pm 0.04^{\circ} \mathrm{C}\right)$ y HR $56.4 \pm 0.6 \%$, durante 14 días después de cosecha (ddc) para su evaluación.

\section{Variables evaluadas}

Se determinó el diámetro polar y ecuatorial de nueve frutos por genotipo. Las mediciones se hicieron cada $7 \mathrm{~d}$ a partir del amarre (10 días después de la floración) hasta su cosecha, con un vernier Truper ${ }^{\circledR}$. Los parámetros de color se midieron con un colorímetro (3nh, NR20XE, Shenzhen, China) en frutos de diferente estado de madurez (USDA, 2005), que registró los valores de luminosidad $(L *)$, cromaticidad $\left(C^{*}\right)$ y matiz $\left(h^{\circ}\right)$. La pérdida de peso (PP) se determinó en \% pesando cinco frutos por genotipo cada tercer día en una balanza digital (Esnova SE-2000, Ciudad de México), mediante la siguiente fórmula: 


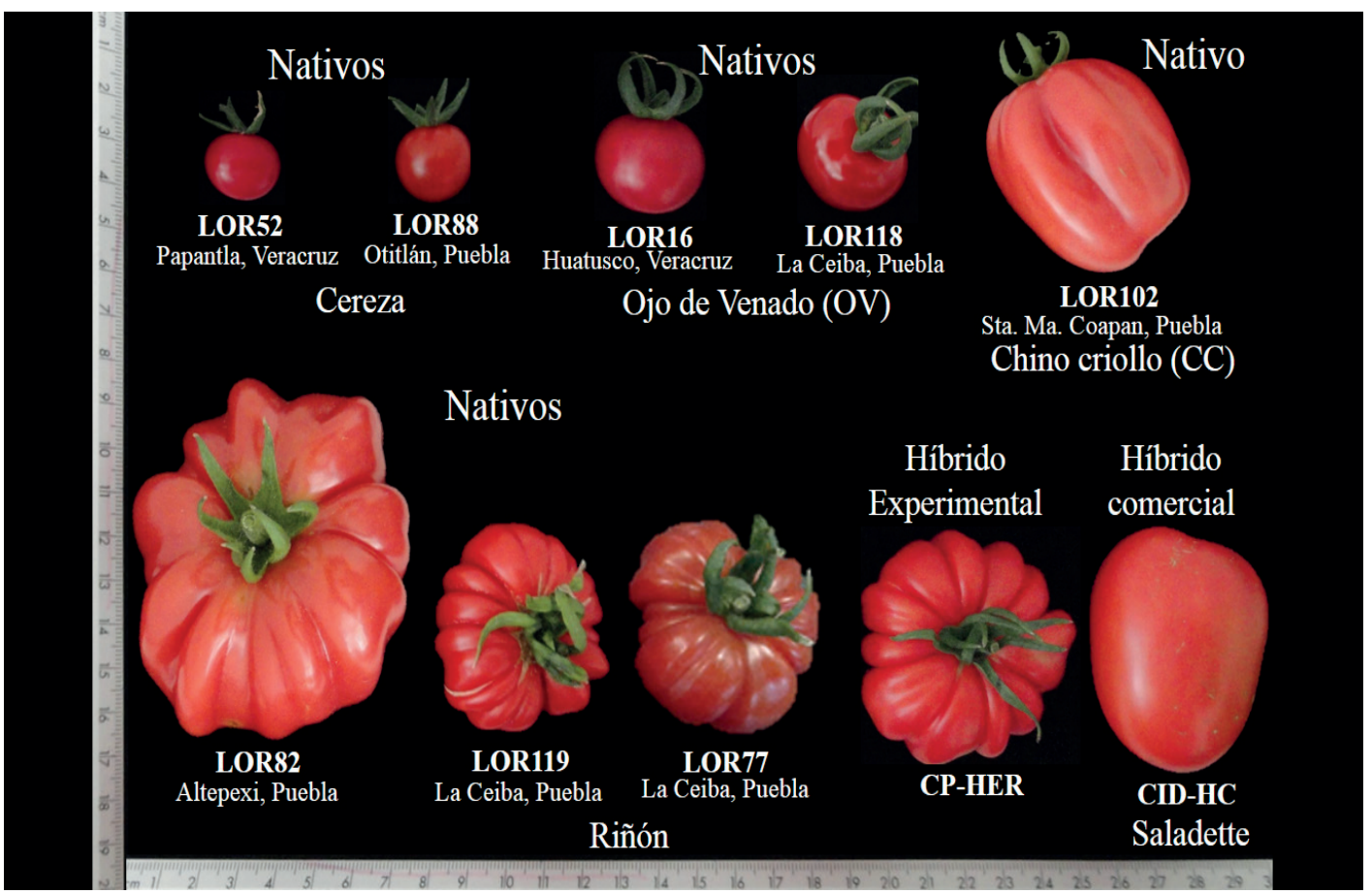

Figura 1. Frutos de tomate (Solanum lycopersicum L.) de genotipos nativos, híbrido experimental e híbrido comercial utilizados en el estudio.

$$
\left.P P=\left(P_{\text {inicial }}-P_{\text {final }}\right) / P_{\text {inicial }}\right) \times 100
$$

La firmeza se determinó con un texturómetro (Force Five $^{\text {TM }}$ FDV-30, Wagner Instruments, Greenwich, Connecticut, EUA) con punzón de 7 mm de diámetro, cuya fuerza para penetrar el fruto se expresó en newtons (N). El contenido de sólidos solubles totales (SST) se midió con un refractómetro (Atago®, Palette PR-32, Tokio, Japón), las lecturas se expresaron en ${ }^{\circ}$ Brix. Para la acidez titulable (AT) se tomaron $5 \mathrm{~g}$ de jugo de los frutos, se colocaron en un matraz Erlenmeyer y se agregaron tres gotas de fenolftaleína, en seguida se tituló con $\mathrm{NaOH} 0.1 \mathrm{~N}$ y se obtuvo el porcentaje de AT con la siguiente formula:

$$
\begin{aligned}
& \% \text { ácido cítrico }=[(\mathrm{V} \mathrm{NaOH}) \times(\mathrm{N} \mathrm{NaOH}) \\
& \times(\text { meq. de ác. cítrico }) \times 100] / \mathrm{g} \text { de jugo }
\end{aligned}
$$

donde: $V$ es el volumen total de jugo y $\mathrm{N}$ es normalidad del $\mathrm{NaOH}$.

Finalmente, se determinó el índice de madurez (IM) con la relación SST/AT (llić et al., 2015). Para el contenido de azúcares solubles totales se tomaron $5 \mathrm{~g}$ de pulpa de tomate, se agregaron $60 \mathrm{~mL}$ de etanol 80 \% y se llevó a ebullición hasta concentrar a $15 \mathrm{~mL}$. Se tomó $1 \mathrm{~mL}$ del concentrado y se pasó por un cartucho de filtración Chromabond (® C18 (Macherey-Nagel, Düren, Alemania) previamente acondicionado. La muestra recuperada se filtró e inyectó en un cromatógrafo de líquidos HPLC (Perkin Elmer 200, Waltham, Massachusetts, EUA) equipado con un detector de índice de refracción (IR), columna Pinnacle II Amino $(150 \times 4.6$ mm, $5 \mu \mathrm{m})$ (Restek®). La fase móvil fue de acetonitrilo/agua $(80: 20 \mathrm{v} / \mathrm{v})$, con un flujo de 1 $\mathrm{mL} / 0.5$ min a $35^{\circ} \mathrm{C}$. La identificación y cuantificación de azúcares se hizo con estándares comerciales (SigmaAldrich, Allentown, Pennsylvania, USA) y los resultados se expresaron en $\mathrm{mg} \mathrm{g}^{-1}$ de peso fresco (pf). Con el contenido de azúcares se obtuvo el índice de dulzor (TSI) con la fórmula utilizada por Beckles (2012):

$$
\begin{gathered}
\text { TSI = }(1.00 \times \text { sacarosa })+(0.76 \times \text { glucosa }) \\
+(1.50 \times \text { fructosa })
\end{gathered}
$$

\section{Diseño experimental y análisis estadístico}

Para las variables de color, PP, firmeza, SST, AT y contenidos de glucosa, fructosa y sacarosa se utilizó un diseño completamente al azar con arreglo factorial $10 \times 2 \times$ 3 con tres repeticiones; los tratamientos se generaron con la combinación de 10 genotipos, dos estados de madurez: rompiente (Estado 1) y rojo (Estado 4 ) y tres periodos de almacenamiento ( 0,7 y en algunos casos $14 \mathrm{~d}$ ); la unidad experimental fue de tres, 10 y 25 frutos, dependiendo del tamaño,

Para comparar el comportamiento del diámetro polar y 
ecuatorial de los frutos se ajustó el modelo logístico $(Y=a / 1$ + be $^{-\mathrm{cx}}$ ) para cada genotipo, donde $Y$ es la variable respuesta (diámetro transversal y polar), $X$ es el tiempo al máximo crecimiento de frutos, $b$ la tasa de crecimiento inicial y $\mathrm{c}$ la tasa de crecimiento utilizando curveExpert 1.4. Para la obtención de las curvas de crecimiento se tomó en cuenta el coeficiente de determinación $\left(R^{2}\right)$ más cercano a 1.0.

Los datos de PP se promediaron y graficaron en función del tiempo y se realizó un análisis de varianza (ANOVA) y se aplicó la prueba de comparación de medias de Tukey $(P \leq 0.05)$. Para el resto de los parámetros (SST, AT, IM, IS, azúcares, y TSI) se aplicó un ANOVA por medio del paquete estadístico R 3.5.0 V. 2018.

\section{RESULTADOS Y DISCUSIÓN}

El crecimiento de los frutos de tomate desde el amarre hasta la etapa de madurez fisiológica fue entre los 44 y $58 \mathrm{~d}$. Los frutos tipo cereza alcanzaron un peso promedio de $3.8 \mathrm{~g}$ por fruto, los frutos OV de $11.2 \mathrm{~g}$, los tipo riñón $68.7 \mathrm{~g}$, los tipo saladette CID-HC $105.0 \mathrm{~g}$ y CC $129.0 \mathrm{~g}$ por fruto (Figura 2). Según Ardila et al. (2011), el crecimiento de los frutos de tomate tiende a seguir una curva sigmoidal; sin embargo, este comportamiento no se ve claramente en algunos materiales, lo que podría atribuirse a que las mediciones fueron muy espaciadas y no se detectaron los cambios en las curvas. Los frutos tipo cereza LOR88 alcanzaron su tamaño final a los 59 d y LOR52 a los 66 días después de la floración (ddf). Los frutos OV LOR16 y LOR118, así como los frutos tipo riñón LOR77 alcanzaron su tamaño máximo a los 66 ddf, seguidos del tipo riñón LOR82, LOR119 y CP-HER, CC LOR102 y el saladette CID-HC, que alcanzaron el tamaño máximo a los $73 \mathrm{~d}$, estos últimos fueron los más tardíos. La madurez de los frutos en la planta, desde el Estado 1 hasta el 4 se logró en aproximadamente 8 d (Figuras 2 y 3). CarrilloRodríguez et al. (2013) reportaron que en colectas de frutos pequeños (tipo cereza) la madurez se alcanza 10 d antes (62.1 ddf) que en los frutos tipo riñón (72.2 ddf) y saladette (70.5 ddf), datos similares a los obtenidos en este trabajo. El crecimiento fue diferencial entre genotipos, ya que cultivares con frutos de menor tamaño requieren menos energía y materia orgánica para su crecimiento que los de mayor tamaño y productividad. Córdoba et al. (2018) reportaron frutos de dos híbridos de tomate cereza Tropical y Tangerino que alcanzaron su tamaño máximo a los 69 y 76 d, respectivamente, en condiciones de invernadero (temperatura entre 14 y $30^{\circ} \mathrm{C}$ y humedad relativa entre 42 y $79 \%$ ), mientras que algunos de los genotipos en el presente estudio fueron más precoces, lo que indica variación genética que puede aprovecharse en programas de mejoramiento genético para precocidad.
Los valores de luminosidad $(L *)$ decrecieron durante la maduración del fruto, con valores de 52 a 65.6 en el Estado 1 de 34.5 a 46.3 en el Estado 4 para todos los genotipos. Estos resultados reflejan el oscurecimiento de los frutos por la degradación de clorofila y la biosíntesis de carotenoides (Arias et al., 2000; Carrillo-López y Yahia, 2014). Por otro lado, los valores de croma aumentaron en todos los genotipos durante la maduración, de 18.2 a 34.7 en el Estado 1 y de 29.5 a 42.0 en el Estado 4, mientras que los valores de hue $\left(h^{\circ}\right)$ disminuyeron durante este periodo y presentaron tonalidades amarillentas-rosadas $\left(h^{\circ}=57\right.$ y 89.6) en el Estado 1, rojas en el Estado $4\left(h^{\circ}=37.4\right.$ y 22) (Figura 3) y presentaron diferencias significativas entre estados de madurez (datos no mostrados).

Los genotipos CC LOR102, CP-HER $\left(1+24.78 e^{-0.097 x}\right)$ y CID-HC cosechados en Estado 1 y los genotipos tipo riñón LOR82, CC LOR102 y CID-HC cosechados en Estado 4 mostraron PP menores o cercanos a $7 \%$ durante el almacenamiento (Figura 4), este valor es deseable para los frutos de tomate, ya que aún no presentan marchitamiento y disminución de la firmeza. La pérdida de agua en tomate ocurre principalmente a través de la cicatriz del pedúnculo y no a través de la cutícula, ya que las células epidérmicas del fruto están cubiertas por capas cutinizadas que forman una barrera física que regula la pérdida de agua (Bouzo y Gariglio, 2016; Juárez-López et al., 2009). No todos los frutos llegaron a los 14 días de almacenamiento (dda) en condiciones aceptables, los frutos del genotipo LOR77 cosechados en Estado 1 sólo alcanzaron 11 días y los frutos OV LOR118, riñón LOR77 y CC LOR102 cosechados en Estado 4 alcanzaron 9 dda; por otro lado, los frutos tipo riñón LOR82, LOR119 y CP-HER alcanzaron 7 días, debido a la alta incidencia de hongos, probablemente por las depresiones naturales en la unión de los carpelos, que mantiene mayor humedad en éstos que en los frutos lisos. En general, los frutos con mayor PP fueron cereza y OV, lo que coincide con lo reportado por Bouzo y Gariglio (2016), quienes señalaron que la PP es mayor en frutos pequeños debido a la mayor área expuesta con respecto a su volumen, lo que conduce a una mayor transpiración.

En relación con la firmeza (Cuadro 1), existen diferencias entre genotipos; los frutos de CID-HC presentaron mayor firmeza, seguidos de CC LOR102 y de los tipo riñón LOR82 en Estado 1 recién cosechados. Estos frutos pueden usarse para la obtención de variedades mejoradas, ya que según Batu (2004), durante el manejo postcosecha los frutos de tomate presentan daño por compresión, vibración e impacto; por lo cual, entre mayor sea la firmeza a la cosecha, mayor resistencia tendrá el fruto a la manipulación durante el transporte y comercialización. En general, los frutos cosechados en Estado 1 perdieron más firmeza durante el almacenamiento que los del Estado 4; ésto se debe a la 


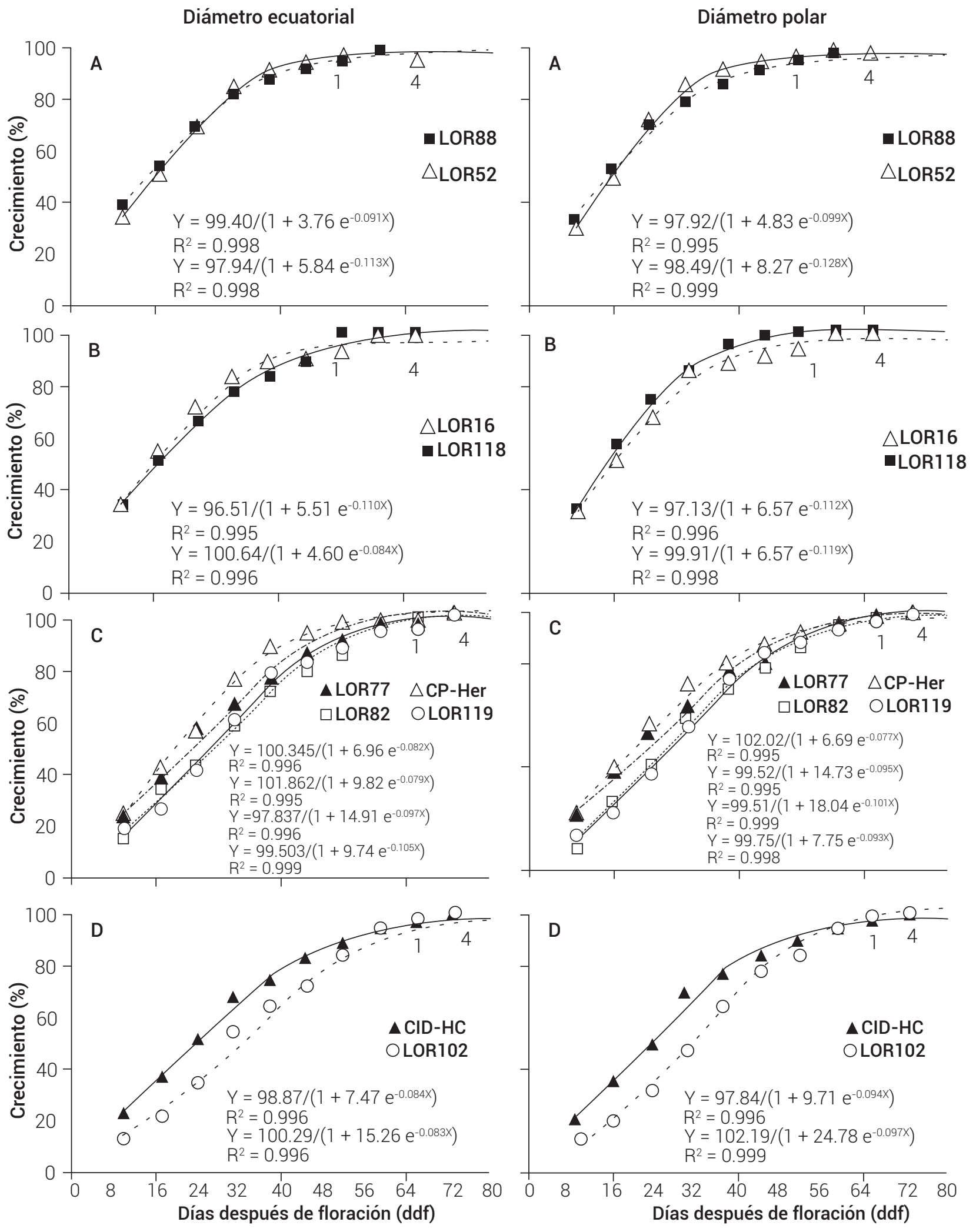

Figura 2. Crecimiento acumulado del diámetro ecuatorial y polar de los frutos de tomate cultivados bajo condiciones de invernadero a distintos días después de la floración (ddf), 1 y 4 indican el estado de madurez rompiente y rojo del fruto, respectivamente. A) genotipos tipo cereza (LOR52 y LOR88), B) OV (LOR16 y LOR118), C) riñón (LOR77, LOR82, LOR1 19 y CP-HER, D) CC (LOR102) y saladette (CID-HC). 


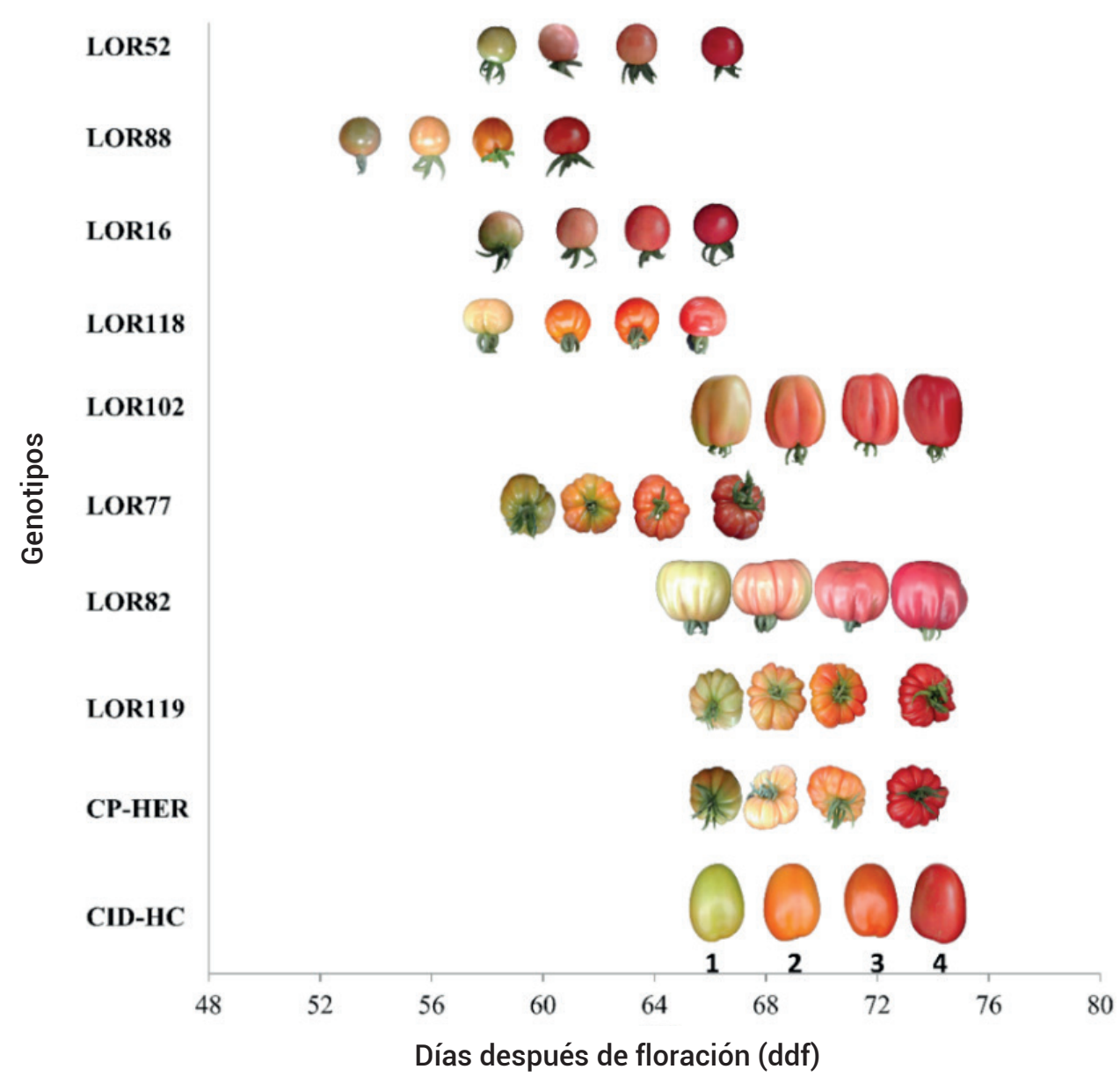

Figura 3. Estados de madurez de los genotipos nativos e híbridos (experimental y comercial) a distintos ddf. Estados de madurez del fruto 1: rompiente, 2: naranja, 3: rojo claro y 4: rojo.

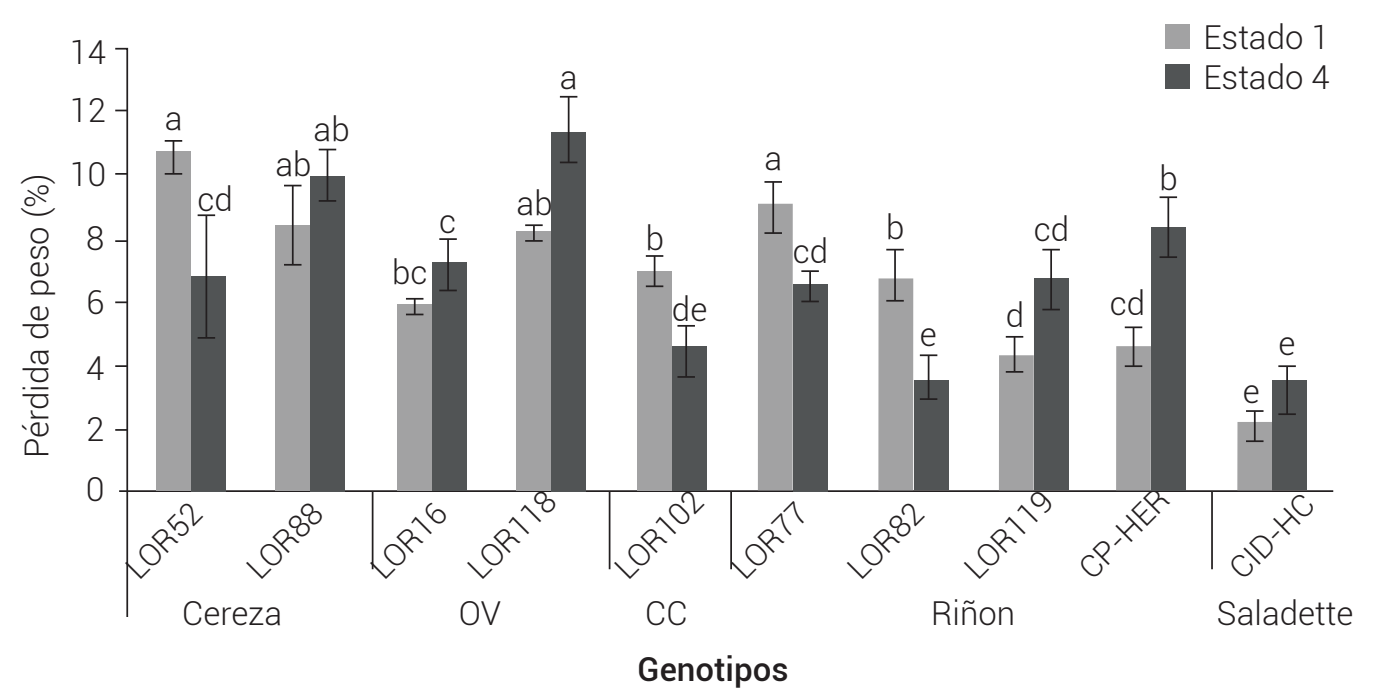

Figura 4. Pérdida de peso (PP) después de $7 \mathrm{~d}$ de almacenamiento a $23.5 \pm 0.04{ }^{\circ} \mathrm{C}$ y $56.4 \pm 0.6 \% \mathrm{HR}$, en frutos de tomate nativos e híbridos cosechados en dos estados de madurez ( 1 y 4). OV: ojo de venado, CC: chino criollo. Valores con diferente letra en cada estado de madurez indican diferencias estadísticamente significativas dentro de cada genotipo (Tukey, $\mathrm{P} \leq 0.05$ ). 
Cuadro 1. Firmeza, sólidos solubles totales, acidez e índice de madurez de genotipos nativos e híbridos de Solanum lycopersicum L.

\begin{tabular}{|c|c|c|c|c|c|c|c|c|c|c|c|}
\hline \multirow{2}{*}{ D.A. } & \multicolumn{2}{|c|}{ Cereza } & \multicolumn{2}{|c|}{ Ojo de venado } & \multirow{2}{*}{$\frac{\text { CC }}{\text { LOR102 }}$} & \multicolumn{4}{|c|}{ Riñón } & \multicolumn{2}{|c|}{ Saladette } \\
\hline & LOR52 & LOR88 & LOR16 & LOR118 & & LOR77 & LOR82 & LOR119 & CP-HER & $\mathrm{CID}-\mathrm{HC}$ & CV (\%) \\
\hline Firmeza (N) & & & & & & Estado 1 & & & & & \\
\hline 0 & $0.63 a$ & $0.97 a$ & $1.14 \mathrm{a}$ & $0.58 a$ & $3.77 \mathrm{a}$ & $1.30 \mathrm{a}$ & $3.01 \mathrm{a}$ & $1.52 \mathrm{a}$ & $1.48 \mathrm{a}$ & $4.44 \mathrm{a}$ & 60.26 \\
\hline 7 & $0.39 b$ & $0.41 b$ & $0.58 b$ & $0.41 b$ & $1.23 b$ & $0.54 b$ & $1.20 \mathrm{~b}$ & $0.69 b$ & $0.61 b$ & $1.22 b$ & 41.02 \\
\hline \multirow[t]{2}{*}{14} & $0.34 b$ & $0.36 b$ & $0.41 \mathrm{c}$ & $0.40 \mathrm{bc}$ & $0.87 \mathrm{c}$ & -- & $0.93 \mathrm{~b}$ & $0.51 \mathrm{~b}$ & $0.44 \mathrm{~b}$ & $0.92 \mathrm{bc}$ & 25.86 \\
\hline & & & & & & Estado 4 & & & & & \\
\hline 0 & $0.42 \mathrm{~b}$ & $0.35 b$ & $0.40 \mathrm{~cd}$ & $0.30 \mathrm{bc}$ & $0.80 \mathrm{c}$ & $0.51 \mathrm{~b}$ & $0.69 c$ & $0.51 \mathrm{~b}$ & $0.45 b$ & $1.20 \mathrm{~b}$ & 25.98 \\
\hline 7 & $0.41 b$ & $0.30 \mathrm{~b}$ & $0.30 \mathrm{~d}$ & $0.29 \mathrm{c}$ & $0.67 \mathrm{c}$ & $0.36 \mathrm{c}$ & $0.59 c$ & $0.47 b$ & $0.38 b$ & $0.87 \mathrm{c}$ & 19.43 \\
\hline 14 & $0.43 b$ & $0.26 b$ & $0.21 \mathrm{e}$ & -- & -- & -- & -- & -- & -- & $0.75 c$ & 19.35 \\
\hline SST ('Brix) & & & & & & Estado 1 & & & & & \\
\hline 0 & $5.98 a$ & $5.95 a$ & $5.16 b$ & $5.36 a$ & $4.36 \mathrm{a}$ & $5.00 \mathrm{~b}$ & $4.36 \mathrm{a}$ & $4.81 \mathrm{a}$ & $4.30 \mathrm{abc}$ & $4.33 a$ & 7.56 \\
\hline 7 & $6.70 \mathrm{a}$ & $5.86 a$ & $6.60 \mathrm{a}$ & $5.25 a b$ & $3.93 \mathrm{a}$ & $3.93 \mathrm{c}$ & 3.93 a & $4.36 a b$ & $4.21 \mathrm{bc}$ & $4.65 a$ & 6.65 \\
\hline \multirow[t]{2}{*}{14} & 6.08 a & $5.71 \mathrm{a}$ & $6.55 a$ & $4.66 \mathrm{~b}$ & 3.88 a & -- & $3.88 \mathrm{a}$ & $3.98 b$ & $3.98 \mathrm{c}$ & $4.18 b$ & 8.87 \\
\hline & & & & & & Estado 4 & & & & & \\
\hline 0 & $6.06 a$ & $5.25 a$ & $5.50 \mathrm{~b}$ & 5.38 a & $4.36 \mathrm{a}$ & $5.81 \mathrm{a}$ & $4.36 \mathrm{a}$ & $4.78 \mathrm{a}$ & $4.86 \mathrm{a}$ & $4.40 \mathrm{a}$ & 6.66 \\
\hline 7 & $5.83 a$ & $5.73 \mathrm{a}$ & $5.25 b$ & $4.96 \mathrm{ab}$ & $4.25 \mathrm{a}$ & $5.41 \mathrm{ab}$ & $4.25 \mathrm{a}$ & $4.75 a$ & $4.65 \mathrm{ab}$ & $4.43 a$ & 8.70 \\
\hline 14 & $5.88 \mathrm{a}$ & $5.60 \mathrm{a}$ & $5.31 \mathrm{~b}$ & -- & -- & -- & -- & -- & -- & $4.20 \mathrm{a}$ & 7.71 \\
\hline AT (\%) & & & & & & Estado 1 & & & & & \\
\hline 0 & 0.78 a & $1.03 \mathrm{a}$ & $0.93 \mathrm{a}$ & 0.68 a & $0.49 a$ & 0.78 a & $0.35 a$ & $0.67 \mathrm{a}$ & $0.59 a$ & $0.43 a b$ & 8.07 \\
\hline 7 & $0.67 b c$ & $0.84 \mathrm{~d}$ & $0.71 \mathrm{~b}$ & $0.69 a$ & $0.42 a b$ & $0.58 \mathrm{~b}$ & $0.40 a$ & $0.62 a b$ & $0.58 \mathrm{a}$ & $0.46 a$ & 7.15 \\
\hline \multirow[t]{2}{*}{14} & $0.60 \mathrm{c}$ & $0.88 \mathrm{~cd}$ & $0.59 c$ & $0.57 b$ & $0.39 \mathrm{~b}$ & -- & $0.40 \mathrm{a}$ & $0.52 b$ & $0.43 b$ & $0.36 c$ & 7.87 \\
\hline & & & & & & Estado 4 & & & & & \\
\hline 0 & $0.67 \mathrm{bc}$ & $0.91 \mathrm{bc}$ & $0.70 \mathrm{~b}$ & $0.67 \mathrm{a}$ & $0.37 b$ & $0.65 b$ & $0.37 \mathrm{a}$ & $0.55 b$ & $0.44 b$ & $0.38 \mathrm{bc}$ & 8.67 \\
\hline 7 & $0.69 b$ & $0.96 b$ & $0.75 b$ & $0.72 \mathrm{a}$ & $0.41 b$ & $0.64 b$ & $0.40 a$ & $0.58 b$ & $0.49 b$ & $0.39 \mathrm{bc}$ & 5.66 \\
\hline 14 & $0.70 \mathrm{~b}$ & $0.87 \mathrm{~cd}$ & $0.72 \mathrm{~b}$ & -- & -- & -- & -- & -- & -- & $0.37 b c$ & 5.99 \\
\hline IM & & & & & & Estado 1 & & & & & \\
\hline 0 & $7.7 b$ & $5.7 \mathrm{a}$ & $5.5 \mathrm{c}$ & $7.9 \mathrm{a}$ & $8.8 \mathrm{~b}$ & $6.4 \mathrm{~b}$ & $12.3 \mathrm{a}$ & $7.2 \mathrm{~b}$ & $7.3 \mathrm{c}$ & $10.0 \mathrm{~b}$ & 4.97 \\
\hline 7 & $10.1 \mathrm{a}$ & $7.0 \mathrm{a}$ & $9.3 \mathrm{~b}$ & $7.6 a$ & $10.0 \mathrm{~b}$ & $6.7 \mathrm{~b}$ & $9.9 \mathrm{c}$ & $7.0 \mathrm{~b}$ & $7.3 c$ & $10.0 \mathrm{~b}$ & 5.35 \\
\hline \multirow[t]{2}{*}{14} & $10.1 \mathrm{a}$ & $6.5 \mathrm{a}$ & $11.1 \mathrm{a}$ & $8.2 \mathrm{a}$ & $10.3 \mathrm{~b}$ & -- & $9.6 \mathrm{c}$ & $7.6 a b$ & $9.1 \mathrm{~b}$ & $11.7 \mathrm{a}$ & 5.32 \\
\hline & & & & & & Estado 4 & & & & & \\
\hline 0 & $9.1 \mathrm{ab}$ & $5.7 \mathrm{a}$ & $7.8 \mathrm{~b}$ & $8.0 \mathrm{a}$ & $12.7 \mathrm{a}$ & 8.9 a & 11.6 ab & $8.7 \mathrm{a}$ & $11.0 \mathrm{a}$ & $11.5 \mathrm{ab}$ & 5.18 \\
\hline 7 & $8.5 b$ & $6.0 \mathrm{a}$ & $7.0 \mathrm{bc}$ & $6.9 \mathrm{a}$ & $9.2 \mathrm{~b}$ & $8.4 \mathrm{a}$ & 10.6 bc & $8.1 \mathrm{ab}$ & $9.4 a b$ & $11.3 \mathrm{ab}$ & 5.31 \\
\hline 14 & $8.4 b$ & $6.4 \mathrm{a}$ & $7.3 \mathrm{bc}$ & -- & -- & -- & -- & -- & -- & $11.2 \mathrm{ab}$ & 5.45 \\
\hline
\end{tabular}

Valores con diferente letra en cada columna indican diferencias estadísticamente significativas entre estados de madurez y tiempo de almacenamiento (Tukey, $\mathrm{P} \leq$ 0.05). D.A.: días de almacenamiento, CV: coeficiente de variación, IM: índice de madurez, --: no alcanzó 14 días de almacenamiento. 
mayor actividad enzimática en los frutos inmaduros que en aquellos que estuvieron en la planta durante el cambio de color. Entre las enzimas más importantes relacionadas con la firmeza se encuentran celulasas, pectinesterasas, enzimas de esterificación y poligalacturonasas, que son promotoras de la solubilización y principales causantes del ablandamiento de los frutos; la expresión de estas enzimas presenta una correlación negativa con la firmeza de los frutos (Sun et al., 2015; Vela-Hinojosa et al., 2018).

Según Batu (2004), los frutos grandes de tomate de calidad comercial (de 50 a $55 \mathrm{~mm}$ de longitud), deben presentar valores de firmeza superiores a $1.45 \mathrm{~N}$ en estado rojo. De acuerdo con los resultados obtenidos, los genotipos que cumplen con este requisito son los tipo riñón LOR82, LOR119, CP-HER, CC LOR102 y CID-HC en Estado 1. Bonilla-Barrientos et al. (2014) reportaron que los frutos de tomate pequeños, como el tipo cereza y OV, generalmente presentan menor firmeza, con un promedio de $0.69 \mathrm{~N}$. En este trabajo, los frutos tipo cereza LOR52 y LOR88 y los del tipo OV LOR16 y LOR 118 tuvieron valores de firmeza de entre 0.30 y $0.42 \mathrm{~N}$, valores inferiores al resto de los frutos.

Con respecto al contenido de SST, no hubo diferencias significativas durante el almacenamiento entre estados de madurez, pero sí entre genotipos. Los frutos de mayor contenido de SST fueron los tipo cereza LOR52 y LOR88, seguidos de los OV LOR16 y LOR118 (Cuadro 1); dichos genotipos registraron valores similares a los reportados por Beckles (2012), quien señala un contenido de $5 \%$ de SST deseable en tomate para consumo en fresco. BonillaBarrientos et al. (2014) reportaron valores de $4.4 \%$ de SST en frutos tipo cereza provenientes de México, mientras que en frutos riñón y saladette reportaron contenidos de 3.88 y $3.99 \%$, respectivamente, valores similares a los encontrados en este estudio.

En cuanto a la AT, ésta disminuyó durante 7 dda en Estado 1, mientras que los frutos en Estado 4 no presentaron cambios significativos durante el almacenamiento. Los genotipos nativos cereza LOR52 y LOR88 y OV LOR16 presentaron los valores más altos de AT en ambos estados, mientras que los híbridos CID-CH y CP-HER tuvieron valores significativamente menores (Cuadro 1). Estos resultados coinciden con reportes que indican que los genotipos nativos presentan mayor acidez (0.50-1.01\%) que los híbridos (0.32-0.72 \%) (George et al., 2004; JuárezLópez et al., 2009). Según Beckles (2012), los principales ácidos orgánicos que contribuyen la acidez son el cítrico y el málico, cuya proporción depende del tipo de tomate; también señala que frutos con valores cercanos al $0.4 \%$ de acidez son considerados deseables para el consumo; en este estudio, los frutos se encontraron dentro de lo reportado por dicho autor. Es importante destacar que los frutos con menor acidez (CC y riñón) fueron aquellos que tuvieron mayor incidencia de hongos durante el almacenamiento, puesto que alimentos con mayor acidez limitan el crecimiento de microorganismos (Rosas-Gallo y López-Malo, 2011).

El sabor de los frutos es una característica relevante de su calidad y está dado por el contenido de azúcares y ácidos orgánicos, además de compuestos volátiles. LoS SST (azúcares, ácidos, pectinas, compuestos fenólicos, entre otros) y la AT son parámetros que se utilizan para medir el sabor de los frutos (Kader, 2008). Los frutos tipo cereza LOR88, OV LOR118 y el híbrido CID-HC no presentaron cambios significativos en el IM (relación ST/AT) durante el almacenamiento en ambos estados de madurez. Los frutos cereza LOR52 y OV LOR16 cosechados en Estado 1 mostraron un incremento significativo en el IM durante el almacenamiento, que coincide con una disminución significativa de la acidez en los frutos. Los frutos tipo riñón LOR77, LOR1 19, CP-HER y CC LOR102 también presentaron un aumento significativo de IM durante su maduración, sin diferencias significativas durante el almacenamiento (Cuadro 1). Hernández et al. (2008) reportaron valores de IM de 9.0 a 9.7 en cultivares de tomate provenientes de Tenerife, España, valores indicadores de sabor adecuado para el consumo. En este estudio se obtuvieron valores similares para los frutos tipo cereza LOR52, CC LOR102, el tipo riñón LOR82 y CID-HC. El IM es un mejor indicador del sabor que los porcentajes de SST y AT por separado, entre mayor sea el IM menor es la acidez de los frutos y mejor el sabor (Beckles, 2012; llić et al. 2015).

Los genotipos cereza LOR52 y LOR88 no presentaron diferencias significativas en contenido de azúcares durante el almacenamiento ni entre estados de madurez (Cuadro 2). Los frutos tipo OV LOR16, LOR118 y CC LOR102 mostraron un aumento significativo $(P \leq 0.05)$ entre estados de madurez. Los frutos tipo riñón LOR77, LOR82 y LOR119 cosechados en Estado 1 disminuyeron significativamente $(P \leq 0.05)$ el contenido de azúcares totales después de $7 \mathrm{dda}$, mientras que seis de los 10 genotipos cosechados en Estado 4 tuvieron un contenido de azúcares significativamente mayor (Cuadro 2). Del contenido de SST, más del 50 \% corresponde a azúcares, los principales fueron fructosa y glucosa. Hernández et al. (2008) estudiaron frutos de tomate de siete cultivares del sur de España, con registros de 8.5 a $10 \mathrm{mg}$ de glucosa $\mathrm{g}^{-1}$ y de 9.6 a $12.4 \mathrm{mg}$ de fructosa $\mathrm{g}^{-1}$, resultados similares a los encontrados en este estudio, donde el azúcar predominante fue fructosa (Cuadro 2).

Con relación al índice de dulzor, los genotipos más dulces fueron los tipo riñón LOR77 y LOR119, con frutos 


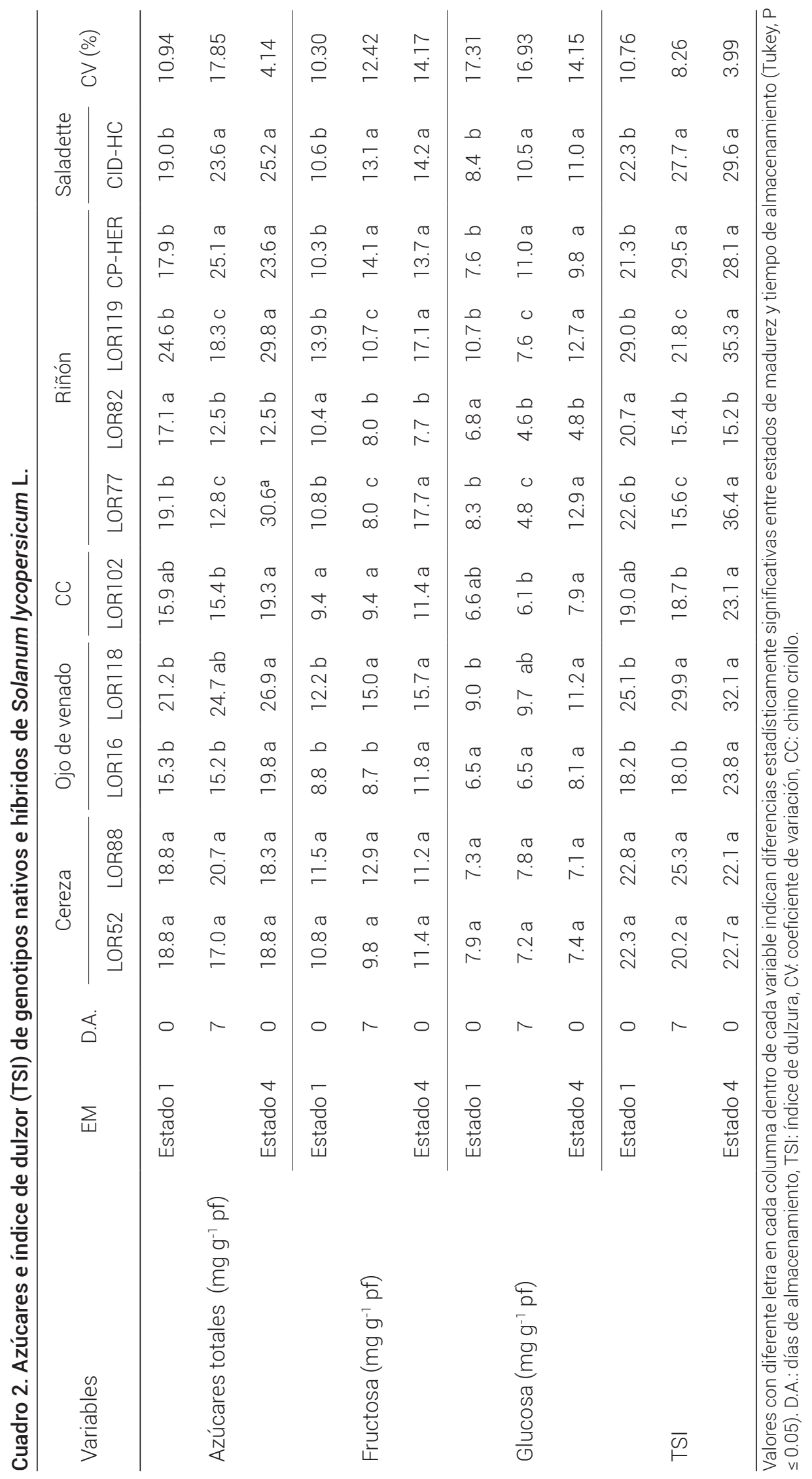


cosechados en Estado 4 y el tipo OV LOR118 y el tipo riñón $\mathrm{CP}-\mathrm{HER}$ con frutos cosechados en Estado 1, de 7 ddc (Cuadro 2). El índice de dulzor involucra a fructosa y glucosa, la concentración y su poder edulcorante (Beckles, 2012).

\section{CONCLUSIONES}

Existen genotipos nativos con potencial para ser usados como fuente de germoplasma en programas de mejoramiento y proporcionar mayor calidad sensorial a los frutos de tomate. Los genotipos cereza LOR88 y riñón LOR77 sobresalen por precocidad dentro de cada grupo. Los genotipos para incrementar dulzor son riñón LOR77 y LOR119, para la acidez el tipo cereza LOR88, con potencial para vida de almacenamiento los tipo cereza, mientras que los de mayor firmeza fueron CC LOR102 y riñón LOR82.

\section{BIBLIOGRAFÍA}

Álvarez-Hernández J. C., H. Cortez-Madrigal e I. García-Ruiz (2009) Exploración y caracterización de poblaciones silvestres de jitomate (Solanaceae) en tres regiones de Michoacán, México. Polibotánica 28:139-159.

Ardila R. G., G. Fischer y H. E. Balaguera-López (2011) Caracterización del crecimiento del fruto y producción de tres híbridos de tomate (Solanum lycopersicum L.) en tiempo fisiológico bajo invernadero. Revista Colombiana de Ciencias Hortícolas 5:4456, https://doi.org/10.17584/rcch.2011v5i1.1252

Arias R., T. C. Lee, L. Logendra and H. Janes (2000) Correlation of lycopene measured by HPLC with the $L * a *, b *$ color readings of a hydroponic tomato and the relationship of maturity with color and lycopene content. Journal of Agricultural and Food Chemistry 48:1697-1702, https://doi.org/10.1021/jf990974e

Bai Y. and P. Lindhout (2007) Domestication and breeding of tomatoes: what have we gained and what can we gain in the future? Annals of Botany 100:1085-1094, https://doi.org/10.1093/aob/mcm150

Batu A. (2004) Determination of acceptable firmness and colour values of tomatoes. Journal of Food Engineering 61:471-475, https://doi.org/10.1016/S0260-8774(03)00141-9

Beckles D. M. (2012) Factors affecting the postharvest soluble solids and sugar content of tomato (Solanum lycopersicum L.) fruit. Postharvest Biology and Technology 63:129-140, https://doi.org/10.1016/j.postharvbio.2011.05.016

Bonilla-Barrientos O., R. Lobato-Ortiz, J. J. García-Zavala, S. Cruz-Izquierdo, D. Reyes-López, E. Hernández-Leal y A. Hernández-Bautista (2014) Diversidad agronómica y morfológica de tomates arriñonados y tipo pimiento de uso local en Puebla y Oaxaca, México. Revista Fitotecnia Mexicana 37:129-139.

Bouzo C. A. and N. F. Gariglio (2016) Relationship between different physical properties of tomato fruits and water loss during postharvest. Acta Scientiarum Polonorum Hortorum Cultus 15:13-25.

Carrillo-López A. and E. M. Yahia (2014) Changes in color-related compounds in tomato fruit exocarp and mesocarp during ripening using HPLC-APCl+-mass spectrometry. Journal of Food Science and Technology 51:2720-2726, https://doi.org/10.1007/s13197-012-0782-0

Carrillo-Rodríguez J. C., J. L. Chávez-Servia, G. Rodríguez-Ortiz, R. Enríquezdel Valle y Y. Villegas-Aparicio (2013) Variación estacional de caracteres agromorfológicos en poblaciones nativas de jitomate (Solanum lycopersicum L.). Revista Mexicana de Ciencias Agrícolas 6:1081-1091.

Córdoba H. A., S. V. Gómez y C. E. Ñústez (2018) Evaluación del rendimiento y fenología de tres genotipos de tomate cherry (Solanum lycopersicum L.) bajo condiciones de invernadero. Revista Colombiana de Ciencias Hortícolas 12:113-125, https://doi.org/10.17584/rcch.2018v12i1.7348

Estrada-Trejo V., R. Lobato-Ortiz, G. García-de los Santos, G. CarrilloCastañeda, F. Castillo-González, E. Contreras-Magaña, O. J. AyalaGaray, M. de la 0 0. y A. Artola M. (2014) Diversidad de poblaciones nativas de jitomate para germinación en condiciones salinas. Revista Mexicana de Ciencias Agrícolas 5:1067-1079.

Figàs M. R., J. Prohens, M. D. Raigón, A. Fita, M. D. García-Martínez, C. Casanova, ... and S. Soler (2015) Characterization of composition traits related to organoleptic and functional quality for the differentiation, selection and enhancement of local varieties of tomato from different cultivar groups. Food Chemistry 187:517-524, https://doi.org/10.1016/j.foodchem.2015.04.083

FIRA, Fideicomisos Instituidos en Relación con la Agricultura (2016) Panorama Agroalimentario. Tomate Rojo 2016. Dirección de Investigación y Evaluación Económica y Sectorial. Fideicomisos Instituidos en Relación con la Agricultura. Ciudad de México. 35 p.

Florido B. M., L. Bao F., R. M. Lara R., M. Álvarez G., F. Dueñas H. y T. Shagarodsky S. (2016) Evaluación de la tolerancia a la sequía en tomate (Solanum lycopersicum) utilizando los índices de tolerancia. Cultivos Tropicales 37:128-135.

George B., C. Kaur, D. S. Khurdiya and H. C. Kapoor (2004) Antioxidants in tomato (Lycopersicum esculentum) as a function of genotype. Food Chemistry 84:45-51, https://doi.org/10.1016/S0308-8146(03)00165-1

Hernández S. M., E. Rodríguez R. and C. Díaz R. (2008) Analysis of organic acid content in cultivars of tomato harvested in Tenerife. European Food Research and Technology 226:423-435 https://doi.org/10.1007/s00217-006-0553-0

Ilić Z. S., L. Milenković, L. Šunić and E. Fallik (2015) Effect of coloured shade nets on plant leaf parameters and tomato fruit quality. Journal of the Science of Food and Agriculture 95:2660-2667, https://doi.org/10.1002/jsfa.7000

Juárez-López P., R. Castro-Brindis, T. Colinas-León, P. Ramírez-Vallejo, M. Sandoval-Villa, D. W. Reed, L. Cisneros-Zeballos y S. King (2009) Evaluación de calidad de frutos de siete genotipos nativos de jitomate (Lycopersicon esculentum var. cerasiforme). Revista Chapingo Serie Horticultura 15:5-9.

Kader A. A. (2008) Flavor quality of fruits and vegetables. Journal of the Science of Food and Agriculture 88:1863-1868, https://doi.org/10.1002/jsfa.3293

Marín-Montes I. M., J. E. Rodríguez-Pérez, J. Sahagún-Castellanos, L. Hernández-Ibáñez and A. M. Velasco-García (2016) Morphological and molecular variation in 55 native tomato collections from Mexico. Revista Chapingo Serie Horticultura 22:117-131, https://doi.org/10.5154/r.rchsh.2016.03.008

Nieto-Ángel D., A. Almaraz-Sánchez, V. Ayala-Escobar, M. OrozcoSantos y F. M. Lara-Viveros (2019) Primer reporte de Colletotrichum coccodes en frutos de tomate (Solanum lycopersicum) en México. Revista Fitotecnia Mexicana 42 195-200, https://doi.org/10.35196/rfm.2019.3.195-200

Peralta I. E., S. Knapp and D. M. Spooner (2006) Nomenclature for wild and cultivated tomatoes. Report of the Tomato Genetics Cooperative 56:6-12.

Rosas-Gallo A. y A. López-Malo (2011) Actividad antimicrobiana del aceite esencial de tomillo (Thymus vulgaris). Temas Selectos de Ingeniería en Alimentos 5:41-50.

Steiner A. A. (1984) The universal nutrient solution. In: Proceedings of the Sixth International Congress on Soilless Culture. 29 April 5 May. Secretariat of International Society for Soilless Culture. Wageningen, The Netherlands. pp:633-650.

Sun Q., N. Zhang, J. Wang, H. Zhang, D. Li, J. Shi, ... Y. D. Guo (2015) Melatonin promotes ripening and improves quality of tomato fruit during postharvest life. Journal of Experimental Botany 66:657-668, https://doi.org/10.1093/jxb/eru332

USDA, United States Department of Agriculture (2005) Tomatoes. Shipping Point and Market Inspection Instructions. Agricultural Marketing Service. United States Department of Agriculture. Washington, D. C. 78 p.

Vásquez-Ortiz R., J. C. Carrillo-Rodríguez y P. Ramírez-Vallejo (2010) Evaluación morfo-agronómica de una muestra del jitomate nativo del Centro y Sureste de México. Naturaleza y Desarrollo 8:49-64. 
Vela-Hinojosa C., C. Barbosa-Martínez, H. B. Escalona-Buendía, J. A. Mendoza-Espinoza, R. Lobato-Ortíz, J. E. Rodríguez-Pérez, J. M. Villa-Hernández and L. J. Pérez-Flores (2018) Architectural diversity of the cuticle and epidermis of native and hybrid tomato fruit genotypes and the relation to polygalacturonase expression. Notulae Botanicae Horti Agrobotanici ClujNapoca 46:45-51 https://doi.org/10.15835/nbha46111001 